\title{
Humoral immune response to Covid-19 vaccination in diabetes: age-dependent but independent of type of diabetes and glycaemic control - the prospective COVAC-DM cohort study
}

Caren Sourij $\mathrm{MD}^{1^{*}}$, Norbert $\mathrm{J}$ Tripolt $\mathrm{PhD}^{2^{*}}$, Faisal Aziz $\mathrm{MSc}^{2^{*}}$, Felix Aberer $\mathrm{MD}^{2,8}$, Patrick Forstner $\mathrm{MD}^{3}$, Anna M Obermayer $\mathrm{MD}^{2}$, Harald Kojzar BSc${ }^{2}$, Barbara Kleinhappl ${ }^{3}$, Peter N Pferschy $\mathrm{MSc}^{2,4}$, Julia K Mader MD ${ }^{5}$, Gerhard Cvirn $\mathrm{MD}^{6}$, Nandu Goswami MD ${ }^{7}$, Nadine Wachsmuth $\mathrm{PhD}^{8}$, Max L. Eckstein ${ }^{8}$, Alexander Müller MSc ${ }^{2}$, Farah Abbas ${ }^{2}$, Jacqueline Lenz ${ }^{2}$, Michaela Steinberger ${ }^{2}$, Lisa Knoll ${ }^{2}$, Robert Krause $M D^{9}$, Martin Stradner $M D^{10}$, Peter Schlenke $M D^{11}$, Nazanin Sareban $\mathrm{MD}^{11}$, Barbara Prietl $\mathrm{PhD}^{4}$, Susanne Kaser $\mathrm{MD}^{12}$, Othmar Moser $\mathrm{PhD}^{2,8}$, Ivo Steinmetz MD ${ }^{3 \#}$, Harald Sourij $\mathrm{MD}^{2 \#}$ on behalf of the COVAC-DM study group

${ }^{1}$ Division of Cardiology, Medical University of Graz, Austria

${ }^{2}$ Interdisciplinary Metabolic Medicine Trials Unit, Division of Endocrinology and

Diabetology, Medical University of Graz, Austria

${ }^{3}$ Institute of Hygiene, Microbiology and Environmental Medicine, Medical University of Graz, Austria

${ }^{4}$ Center for Biomarker Research in Medicine (CBmed), Graz, Austria

${ }^{5}$ Division of Endocrinology and Diabetology, Medical University of Graz, Austria

${ }^{6}$ Division of Physiological Chemistry, Medical University of Graz, Austria

${ }^{7}$ Division of Physiology, Otto Loewi Research Center, Medical University of Graz, Graz, Austria

${ }^{8}$ Division of Exercise Physiology and Metabolism, Institute of Sport Science, Bayreuth, Germany

${ }^{9}$ Division for Infectious Diseases, Medical University of Graz, Graz, Austria 
${ }^{10}$ Division of Rheumatology and Immunology, Medical University of Graz, Austria

${ }^{11}$ Department of Blood Group Serology and Transfusion Medicine, Medical University

of Graz, Austria

${ }^{12}$ Department for Internal Medicine I, Medical University of Innsbruck, Austria

*contributed equally first author

"contributed equally last author

Corresponding Authors: Professor Harald Sourij, MD

Auenbruggerplatz 15,

8036 Graz, Austria

Tel.: +4331638581310

Fax.:

Email address: ha.sourij@medunigraz.at

Word count: 3072

Number of Tables: 1

Number of Figures: 3 


\section{Aims:}

Immune response to COVID-19 vaccination and a potential impact of glycaemia on antibody levels in people with diabetes remains unclear. We investigated the seroconversion following first and second COVID-19 vaccination in people with type 1 and type 2 diabetes in relation to glycaemic control prior to vaccination and analysed the response in comparison to individuals without diabetes.

\section{Materials and Methods:}

This prospective, multicenter cohort study analysed people with type 1 and type 2 diabetes, well $(\mathrm{HbA} 1 \mathrm{c}<7.5 \%$ or $<58 \mathrm{mmol} / \mathrm{mol})$ or insufficiently $(\mathrm{HbA} 1 \mathrm{c} \geq 7.5 \%$ or $\geq 58$ $\mathrm{mmol} / \mathrm{mol}$ ) controlled and healthy controls. Roche's Elecsys anti-SARS-CoV-2 S was used to quantify anti-spike protein antibodies 7-14 days after the first and 14-21 days after the second vaccination.

\section{Results:}

86 healthy controls and 161 participants with diabetes were enrolled, 150 (75 with type 1 diabetes and 75 with type 2 diabetes) were eligible for the analysis. After the first vaccination, only $52.7 \%$ in the type 1 diabetes group and $48.0 \%$ in the type 2 diabetes group showed antibody levels above the cut-off for positivity. Antibody levels after the second vaccination were similar in people with type1, type 2 diabetes and healthy controls if adjusted for age, sex and multiple testing ( $p>0.05)$. Age ( $r=-$ $0.45, p<0.001)$ and glomerular filtration rate $(r=0.28, p=0.001)$ were significantly associated with antibody response.

\section{Conclusions:}

Anti-SARS-CoV-2 S antibody levels after the second vaccination were comparable in healthy controls, people with type 1 and type 2 diabetes, irrespective of glycaemic 
medRxiv preprint doi: https://doi.org/10.1101/2021.11.05.21265849; this version posted November 9, 2021. The copyright holder for this preprint (which was not certified by peer review) is the author/funder, who has granted medRxiv a license to display the preprint in perpetuity.

It is made available under a CC-BY-ND 4.0 International license .

control. Age and renal function correlated significantly with the extent of antibody levels. 


\section{Introduction:}

After the first occurrence of the SARS-CoV-2 virus causing the coronavirus disease (COVID-19) in China in December 2019, the virus has rapidly spread globally leading to the declaration of COVID-19 a pandemic in March 2020 by the World Health Organization (WHO) ${ }^{1}$. Reports from China ${ }^{2},{ }^{3}$, Europe ${ }^{4}$ and the USA ${ }^{5}$ demonstrated that the prevalence of diabetes is as high as $20 \%$ in people hospitalized for COVID-19. Moreover, diabetes is frequent in people experiencing a severe or fatal disease course of COVID- $19^{6}$ indicating an in-hospital mortality to be as high as $25 \%$ in people with diabetes mellitus. ${ }^{7}$

The reasons for the severity of COVID-19 in people with diabetes mellitus are complex; however, in general metabolic diseases including type 2 diabetes present with chronic, systemic low-grade inflammation ${ }^{8}$. This leads to exaggerated cytokine release, inflammation, impaired phagocytosis or glycation of immunoglobulins ${ }^{9}$ potentially altering the outcome of people with diabetes mellitus being exposed to infection. Furthermore, clearing of SARS-CoV-2 requires an effective response of the adaptive immune system. People with obesity or type 2 diabetes have pre-existing alterations in the adaptive immune system ( $B$ and $T$ lymphocytes) including $T$ cells expressing lower levels of co-stimulatory molecules (CD69, CD28, CD40 ligand) or interleukin-12 receptor, resulting in a reduced production of interferon and granzyme B compared to people without type 2 diabetes ${ }^{10,11}$.

Therefore, people with diabetes are usually considered as high-risk population for experiencing adverse COVID-19 outcomes and consequently, COVID-19 vaccination is highly recommended in this population leading to prioritization in current vaccination strategies of most countries ${ }^{12}$. Given the fact that a compromised immune response to SARS-CoV-2 is discussed as a reason for the increased risk for 
severe COVID-19 in people with diabetes, there also remains the question whether people with diabetes do also face a reduced immune response following SARS-CoV2 vaccinations. While most studies on hepatitis $B$ vaccination have demonstrated a reduced immunogenicity in people with diabetes ${ }^{13}$, data on other vaccinations including those against influenza, varicella zoster virus or pneumococcus were either inconclusive or remain lacking ${ }^{13}$.

While phase III studies on both, mRNA and adenovirus based COVID-19 vaccines have included people with diabetes and the efficacy rates in people with diabetes appear to be similar to those of their counterparts without diabetes ${ }^{14-17}$, little data is provided on the characteristics of included people with diabetes. Recently a study suggested lower antibody levels in response to COVID-19 vaccination in people with diabetes. However the number of people with diabetes included was limited with no differentiation between type 1 and type 2 diabetes and without details of the a potential impact of glycaemic control prior to receiving the vaccine ${ }^{18}$.

Therefore, we investigated the humoral immune response and side effects related to COVID-19 vaccines in people with type 1 and type 2 diabetes to elucidate the impact of the type of diabetes and of glycaemic control on antibody response following COVID-19 vaccinations. Moreover, we aimed to compare SARS-CoV-2 antibody levels after COVID-19 vaccination in people with diabetes to healthy, non-diabetes controls.

\section{Materials and Methods:}

The "Immune response to Covid-19 vaccination in people with Diabetes Mellitus COVAC-DM" study was a prospective, multicenter, real world, cohort study including 161 individuals with diabetes mellitus at two centers in Austria (Medical University of 
Graz and Medical University of Innsbruck) and one center in Germany (University of Bayreuth). We included adults with type 1 or type 2 diabetes, aged 18-80 years, who were diagnosed with diabetes prior to receiving a COVID-19 vaccine and willing to give written informed consent. Main exclusion criteria were: active malignancy (excluding intraepithelial neoplasia of the prostate gland and the gastrointestinal tract and basalioma), pregnancy, acute inflammatory disease, immunosuppressant therapy, alcohol abuse (more than 15 standard drinks a week) or any contraindication to the vaccine as well as a previous episode of COVID-19.

People with established type 1 or type 2 diabetes and planned COVID-19 vaccination were recruited from outpatient clinics at the participating sites, from the Graz Diabetes Registry for Biomarker Research and advertisements in local newspapers.

Participants were then enrolled according to their HbA1c and type of diabetes into one of the four predefined groups: well controlled type 1 diabetes with an $\mathrm{HbA1c}$ $\leq 7.5 \%(\leq 58 \mathrm{mmol} / \mathrm{mol})$, insufficiently controlled type 1 diabetes with an $\mathrm{HbA} 1 \mathrm{c}>7.5 \%$ (>58mmol $/ \mathrm{mol})$, well controlled type 2 diabetes with an $\mathrm{HbA} 1 \mathrm{c} \leq 7.5 \%(\leq 58 \mathrm{mmol} / \mathrm{mol})$ and insufficiently controlled type 2 diabetes with an $\mathrm{HbA} 1 \mathrm{c}>7.5 \%(>58 \mathrm{mmol} / \mathrm{mol})$.

All participants were asked to attend on-site visits 60 to two days prior to their first vaccination, 7 to 14 days after their first vaccination and 14 to 21 days after their second vaccination. A physical examination was performed, blood samples were taken and saliva samples for further analyses were collected at each visit. Data on medical history and medication was collected at baseline and information about side effects to vaccination including severe allergic reaction, local injection site reaction (swelling, redness, pain), elevated body temperature between $37^{\circ}$ and $38^{\circ} \mathrm{C}$ or body temperature $>38^{\circ} \mathrm{C}$, headache, arthralgia, fatigue, or hospitalization within 14 days 
after vaccination were recorded at all follow up visits. Biobank samples (serum, plasma, saliva, and peripheral blood mononuclear cells) are stored at $-80^{\circ} \mathrm{C}$ at the

Biobank Graz of the Medical University Graz for further analysis. Antibody tests were conducted at the Institute of Hygiene, Microbiology and Environmental Medicine at the Medical University of Graz. A CE-marked serological test was used according to the manufacturers' protocols to determine and quantify specific antibodies against SARS-CoV-2. Total immunoglobulin (lg) was determined by using the Roche Elecsys anti-SARS-CoV-2 S electrochemiluminescence immunoassay targeting the receptor-binding domain of the viral spike protein using a cobas e 801 analytical unit (Roche Diagnostics $\mathrm{GmbH}$, Mannheim, Germany). Its quantification range lies between 0.4 and $2500 \mathrm{U} / \mathrm{mL}$, while the cut-off for positivity is $0.8 \mathrm{U} / \mathrm{mL}$. According to Roche's protocol ${ }^{19}$ no converting factor is needed to calculate binding antibody units (BAU) per milliliter, which were retrospectively established for harmonization of different assays' results and are traceable to the WHO international standard for antiSARS-CoV-2 $\lg ^{20}$.

In addition, antibody levels, measured 14 to 21 days after the second COVID-19 vaccination from a cohort of 86 healthy people recruited in a partner study (EudraCT: 2021-001040-10) at the Medical University of Graz were used for group comparisons.

The study protocol was approved by the ethics committees of the Medical University of Graz (33-366 ex 20/21) and the Bayerische Landesaerztekammer (Nr. 21031) as well as registered at the European Union Drug Regulation Authorities Clinical Trials registry (EudraCT-Number 2021-001459-15). The study was conducted according to the guidelines of Good Clinical Practice and the Declaration of Helsinki. Prior to 
study inclusion, participants were informed about all study procedures by a physician and provided their written informed consents.

\section{Statistical analysis}

Data were extracted in Microsoft Excel and analysed in Stata version 16 and $R$ studio 1.4.1. Categorical variables were summarized as frequencies and percentages (\%). Quantitative variables were summarized as means and standard deviations $( \pm S D)$. Categorical variables were compared with diabetes groups using Chi-squared or Fisher's exact tests as appropriate. Quantitative variables were compared with diabetes groups using one-way ANOVA tests. Post-vaccination side effects were compared between people with type 1 diabetes and type 2 diabetes using Chi-squared or Fisher's exacts tests as appropriate. Anti-SARS-CoV-2 S antibodies were summarized as median with interquartile range (IQR). The median anti-SARS-CoV-2 S antibody levels were compared between diabetes groups and healthy controls using Kruskal-Wallis tests. These group comparisons were adjusted for age and sex using quantile regression and further adjusted for multiple comparisons using post-hoc Bonferroni correction, or Wilcoxon signed- rank test, respectively. The correlation of anti-SARS-CoV-2 S antibodies with quantitative variables was assessed using Pearson correlation method. The p-value of $<0.05$ was chosen to decide statistical significance.

\section{Results:}

We enrolled 161 participants with diabetes between April and June 2021 in the study, of whom 150 were included in the final analysis (Supplemental Figure 1). Two participants withdrew consent, six participants decided to postpone their vaccination 
for a longer period after the baseline visit and three people had positive anti-SARSCoV-2 S antibodies at baseline without having recognized a COVID-19 episode before. (Supplemental Figure 1). Seventy-five participants had type 1 diabetes (34 females) with 49 being in the well controlled group, having a mean $\mathrm{HbA} 1 \mathrm{c}$ of $6.6 \pm$ $0.6 \%(49 \pm 7 \mathrm{mmol} / \mathrm{mol})$ and 26 being insufficiently controlled with a mean $\mathrm{HbA} 1 \mathrm{c}$ of $8.4 \pm 0.9 \%(68 \pm 10 \mathrm{mmol} / \mathrm{mol})$. In addition, 75 people with type 2 diabetes (34 females) of whom 37 had well controlled diabetes (mean HbA1c $6.5 \pm 0.6 \%$ (48 \pm 7 $\mathrm{mmol} / \mathrm{mol})$ ) and 38 insufficiently controlled diabetes (mean HbA1c $8.4 \pm 0.9 \%(68 \pm 9$ $\mathrm{mmol} / \mathrm{mol}$ )) were included in the study. People with type 2 diabetes were older as compared to those with type 1 diabetes $(56.6 \pm 9.9$ vs. $41.5 \pm 14.5$ years, $p<0.001)$ and had higher prevalence of hypertension, hyperlipidemia, liver diseases and polyneuropathy (for all $\mathrm{p}<0.05$ ). $86 \%$ of all participants received the BioNTech/Pfizer, 8.7\% the Moderna and 5.3\% the AstraZeneca vaccine. Vaccine distribution was similar in all four groups of people with diabetes $(p=0.542)$. A full list of baseline characteristics according to the four groups of study participants is provided in table 1.

Healthy control group:

For comparison we used a cohort of 86 healthy participants. 49 (57\%) were female and the mean age was $48 \pm 11.6$ years. $96.5 \%$ of them received the Moderna and 3.5\% the BioNTech/Pfizer vaccine.

\section{Side effects of vaccination}

Three cases of hospitalization occurred after the vaccination. One occurred 24 days after the first vaccination due to peripheral edema and chronic heart failure. The second 12 days after the second vaccination due to atrioventricular block grade 3 
with subsequent pacemaker implantation and the third hospitalization occurred due to a miscarriage after 10 weeks of pregnancy. Conception was estimated at 2 weeks after the first vaccination. No cases of severe allergic reactions were recorded throughout the study. The most common side effects were injection site reactions, in $87.4 \%$ of all participants after the first and $63.3 \%$ after the second dose, with a significantly lower rate in people with type 2 diabetes at the latter vaccination. Headache was present in $28 \%$ and fatigue in approximately one third of all participants after both injections. Fever was rarely reported in all groups (for detailed overview see Figure 1).

\section{Antibody response}

Seven to 14 days after the first vaccination, $52.7 \%$ of those with type 1 diabetes and $48.0 \%$ of those with type 2 diabetes had anti-SARS-CoV2-S antibodies above the detection limit of 0.8, with low median levels of 1.1 (IQR 8.1) and 0.3 (IQR 2.4), respectively. When we analysed the antibody data measured after the second vaccination, we first pooled both groups of participants with type 1 diabetes and the two groups of participants with type 2 diabetes (well and insufficiently controlled), respectively, and compared against the healthy controls. In the unadjusted analyses we observed the highest antibody levels after second vaccination in people with type 1 diabetes ( $p=0.022$ vs. healthy controls and $p=0.013$ vs. people with type 2 diabetes) (Supplemental Figure 2). However, these significant differences were not further present after adjustment for age, sex and multiple comparisons (Figure 2A). In addition, we investigated the impact of type of diabetes and glycaemic control on antibody response after COVID-19 vaccination. In the group comparison adjusted for multiple comparisons only, people with well controlled type 1 diabetes had 
numerically albeit not statistically significant higher antibody levels as compared to people with insufficiently controlled type 1 diabetes $(p=0.249)$. In comparison to people with well or insufficiently controlled type 2 diabetes, those with well controlled type 1 diabetes had significantly higher antibody levels $(p=0.034$ and $p=0.003$, respectively). After adjusting for age, sex and multiple comparisons, no significant difference between the four groups was observed (Figure 2B).

\section{Clinical characteristics and antibody response}

We pooled all people with diabetes to perform correlation analyses, in which age was moderate-to-strongly correlated with anti-SARS-CoV-2 S antibody levels ( $r=-$ $0.45, p<0.001)$, an association, that was more pronounced in people with type 1 diabetes $(-0.53, p<0.001)$ than in type 2 diabetes patients $(r=-0.20, p=0.087)$. The estimated glomerular filtration rate (eGFR) was also directly associated with levels of anti-SARS-CoV-2 S antibodies $(r=0.28, p=0.001)$, while no correlation was observed with either $\mathrm{HbA1c}$ levels at baseline $(r=-0.07, p=0.398)$ or with changes of $\mathrm{HbA1c}$ levels between baseline and the follow up visit after the second vaccination $(r=0.06$, $\mathrm{p}=0.509$ ) as a measure of change in glycaemic control between the vaccinations. Body mass index was weakly and inversely correlated with humoral immune response $(r=-0.18, p=0.027)$. Gender and diabetes duration had no impact on the antibody response.

If participants developed an elevated body temperature ( $>37.0$ degree Celsius) after the second vaccination, the antibody response appeared to be more pronounced $(p=0.036)$ as compared to those without such an increase in body temperature (Supplemental Figure 5). 


\section{Discussion:}

Our study demonstrated that people with type 1 and type 2 diabetes display a humoral immune response to COVID-19 vaccination measured by anti-receptor binding domain SARS-CoV-2 S antibodies, that is comparable to healthy controls. While unadjusted analyses suggested higher antibody levels in people with well controlled type 1 diabetes, this difference does not persist after adjustment for age, sex and multiple comparisons. Our data also suggest that age and estimated glomerular filtration rate are predictors for antibody levels after COVID-19 vaccination, while $\mathrm{HbA1c}$ levels are not.

Our study results are in contrast to a recent observational study from Italy (CAVEAT study) that demonstrated a lower antibody response to COVID-19 vaccination in people with type 2 diabetes having an $\mathrm{HbA} 1 \mathrm{c}$ above $7.0 \%$ (53 $\mathrm{mmol} / \mathrm{mol})$. This was accompanied by a reduced CD4pos $T$ cell response measured by tumor necrosis factor- $\alpha$, interleukin-2 or interferon- $\gamma$ response ${ }^{21}$. In contrast to our study the Italian study did not prospectively prespecify their diabetes cohort and $\mathrm{HbA} 1 \mathrm{c}$ cut-off for the analyses according to the given registration record (NCT04746521). Although we predefined a cut-off of $7.5 \%(58 \mathrm{mmol} / \mathrm{mol})$ to separate well from insufficiently controlled people with diabetes, the mean $\mathrm{HbA1c}$ levels observed in our cohort in the two groups (well and insufficiently controlled) are comparable to those in the Italian study. Another difference between the studies is the antibody assays used; while the CAVEAT study used the GenScript SARS-CoV-2 surrogate virus neutralization test we used the Roche Elecsys anti-SARS-CoV-2 S, that was also shown to correlate with neutralizing antibodies ${ }^{22}$. Hence, in direct comparison studies, both assays have demonstrated good correlation with each other with an agreement rate of approximately $90 \%{ }^{23}$. In contrast to our study, Marfella et al. did not include people 
with type 1 diabetes ${ }^{21}$, which is however of clinical relevance as it was unclear if the immune response to COVID-19 vaccination is determined by the underlying pathophysiology of diabetes.

Our study clearly suggests that age is a major determinant of humoral immune response to a COVID-19 vaccination. Previous studies have shown that elderly people not only exhibit a lower antibody response to different types of vaccines like diphtheria, hepatitis $A$, hepatitis $B$, pneumococcal polysaccharide vaccine, tick-borne encephalitis, tetanus or trivalent influenza vaccine, they also display a more rapid waning of antibodies ${ }^{24}$.

Besides age, other clinical features seem to predict antibody response: Mingyao Ma et al. described in their review, that seroconversion rates after HBV-vaccination decreases with lower kidney function from $95 \%$ in healthy subjects to $40-50 \%$ in people with CKD stage 3 to $4^{25}$. Likewise, the estimated glomerular filtration rate in our study was directly associated with the level of anti-SARS-CoV-2 S antibodies, suggesting that re-vaccination intervals in people with diabetes and advanced diabetic kidney disease might need to be shorter.

As in previous studies with hepatitis vaccines ${ }^{26}$, we could also show a significant inverse association of the body mass index with anti-SARS-CoV-2 S antibodies. However, in contrast to the hepatitis vaccination study, the correlation in our dataset was rather weak $(r=-0.19, p=0.027)$. Also, no correlation was found with diabetes duration in our study $(r=-0.06, p=0.495)$.

Our study is not without limitations. We aimed to recruit 40 participants into each subgroup of people with diabetes, a number which, despite large efforts, was not reached for those with type 1 diabetes and insufficient glycaemic control. In addition, in our study we focused on humoral immune response only and did not further 
investigate the cellular immune response after the vaccination. However, previous studies have clearly shown, that neutralizing antibody levels are highly predictive of immune protection from symptomatic SARS-CoV-2 infections ${ }^{27}$. As our study is still ongoing with follow up visits planned before and after a potential $3^{\text {rd }}$ vaccination and / or 12 months after the baseline visit, data on cellular immune response will be available at future visits.

While measurement of neutralizing antibody testing is the gold standard, it is time and resource consuming. Hence commercial assays such as the Roche Elecsys anti-SARS-CoV-2 S electrochemiluminescence immunoassay targeting the receptorbinding domain of the viral spike protein used in our study has proven to be a reliable surrogate measurement for neutralizing anti-SARS-CoV-2 antibodies ${ }^{28}$.

Another limitation of our study is that we included participants from two Austrian sites and one German site only, representing a white Caucasian population - immune response to COVID-19 vaccination should clearly be replicated in other ethnic groups and for other vaccines, as we included 142 subjects $(94.6 \%)$ receiving an mRNA-based vaccine. However, since we performed a real-world cohort study within the national vaccination strategy of Austria and Germany the observed distribution of vaccines represents the actual distribution in these countries in people with diabetes. The COVAC-DM study demonstrated similar humoral immune response to COVID19 vaccination in people with type 1 and type 2 diabetes and healthy controls, when results were adjusted for age, which, together with renal function, has a significant impact on antibody response in our study. Additional follow up in our study and other clinical trials will help to clarify the trajectories of antibody levels after COVID-19 vaccination in people with diabetes and the re-vaccination intervals depending on patient characteristics. 


\section{Acknowledgements:}

Author contributions

HS, NJT, FAz, IS and CS designed the study. CS and HS drafted the first version of the manuscript. HK, PNP, NW and NJT performed the data preparation. FAz performed statistics and created the figures. CS, FAb, AMO, HK, PNP, ML, JKM MLE, AM, JL, FAbb, GC, NG, MS, LK, NS, performed the subject recruitment and were in charge of the conduction of study visits. BK, PF and IS performed antibody measurements. IS supervised antibody measurements. BP performed lab measurements. RK, MSt, PS supervised and designed the healthy control study. Data on healthy subjects was provided by MS. SK acted as principal investigator of the participating study center in Innsbruck. OM was responsible for the performance of the study at the center in Bayreuth. All of the authors have carefully revised the manuscript, agreed to the submission of the latest version and sufficiently contributed to this work. The samples/data used for this project have been provided by Biobank Graz of the Medical University of Graz, Austria."

All authors critically revised the manuscript.

We would like to thank Marlies Leitner, Lejla Pesto, Harald Rupprecht, Beate Zunner, Sandra Haupt and Tamara Banfic for their support.

Conflict-of-interest statement:

All authors have no conflicts of interest to declare.

\section{Funding:}

This research received no specific grant from any funding agency in the public, commercial or non-for-profit sectors. However, funding was applied for at the Austrian Science Fund. 


\section{References}

1. Cucinotta D, Vanelli M. WHO Declares COVID-19 a Pandemic. Acta Biomed. 2020;91(1):157-160.

2. Guan WJ, Ni ZY, Hu Y, et al. Clinical Characteristics of Coronavirus Disease 2019 in China. N Engl J Med. 2020;382(18):1708-1720.

3. Zhou F, Yu T, Du R, et al. Clinical course and risk factors for mortality of adult inpatients with COVID-19 in Wuhan, China: a retrospective cohort study. Lancet. 2020;395(10229):1054-1062.

4. Grasselli G, Zangrillo A, Zanella A, et al. Baseline Characteristics and Outcomes of 1591 Patients Infected With SARS-CoV-2 Admitted to ICUs of the Lombardy Region, Italy. JAMA. 2020;323(16):1574-1581.

5. Richardson S, Hirsch JS, Narasimhan M, et al. Presenting Characteristics, Comorbidities, and Outcomes Among 5700 Patients Hospitalized With COVID-19 in the New York City Area. JAMA. 2020;323(20):2052-2059.

6. $\mathrm{Wu} \mathrm{Z,} \mathrm{McGoogan} \mathrm{JM.} \mathrm{Characteristics} \mathrm{of} \mathrm{and} \mathrm{Important} \mathrm{Lessons} \mathrm{From} \mathrm{the}$ Coronavirus Disease 2019 (COVID-19) Outbreak in China: Summary of a Report of 72314 Cases From the Chinese Center for Disease Control and Prevention. JAMA. 2020;323(13):1239-1242.

7. Sourij H, Aziz F, Brauer A, et al. COVID-19 fatality prediction in people with diabetes and prediabetes using a simple score upon hospital admission. Diabetes Obes Metab. 2021;23(2):589-598.

8. Ryan PM, Caplice NM. Is Adipose Tissue a Reservoir for Viral Spread, Immune Activation, and Cytokine Amplification in Coronavirus Disease 2019? Obesity (Silver Spring). 2020;28(7):1191-1194.

9. Perez-Galarza J, Procel C, Canadas C, et al. Immune Response to SARS-CoV-2 Infection in Obesity and T2D: Literature Review. Vaccines (Basel). 2021;9(2).

10. Xia C, Rao X, Zhong J. Role of T Lymphocytes in Type 2 Diabetes and DiabetesAssociated Inflammation. J Diabetes Res. 2017;2017:6494795.

11. DeFuria J, Belkina AC, Jagannathan-Bogdan M, et al. B cells promote inflammation in obesity and type 2 diabetes through regulation of T-cell function and an inflammatory cytokine profile. Proc Natl Acad Sci U S A. 2013;110(13):5133-5138.

12. Powers AC, Aronoff DM, Eckel RH. COVID-19 vaccine prioritisation for type 1 and type 2 diabetes. Lancet Diabetes Endocrinol. 2021;9(3):140-141.

13. Verstraeten T, Fletcher MA, Suaya JA, et al. Diabetes mellitus as a vaccine-effect modifier: a review. Expert Rev Vaccines. 2020;19(5):445-453.

14. Polack FP, Thomas SJ, Kitchin N, et al. Safety and Efficacy of the BNT162b2 mRNA Covid-19 Vaccine. N Engl J Med. 2020;383(27):2603-2615.

15. Baden LR, El Sahly HM, Essink B, et al. Efficacy and Safety of the mRNA-1273 SARS-CoV-2 Vaccine. N Engl J Med. 2021;384(5):403-416.

16. Voysey M, Costa Clemens SA, Madhi SA, et al. Single-dose administration and the influence of the timing of the booster dose on immunogenicity and efficacy of ChAdOx1 nCoV-19 (AZD1222) vaccine: a pooled analysis of four randomised trials. Lancet. 2021;397(10277):881-891.

17. Voysey M, Clemens SAC, Madhi SA, et al. Safety and efficacy of the ChAdOx1 nCoV-19 vaccine (AZD1222) against SARS-CoV-2: an interim analysis of four randomised controlled trials in Brazil, South Africa, and the UK. Lancet. 2021;397(10269):99-111. 
18. Lustig Y, Sapir E, Regev-Yochay G, et al. BNT162b2 COVID-19 vaccine and correlates of humoral immune responses and dynamics: a prospective, single-centre, longitudinal cohort study in health-care workers. Lancet Respir Med. 2021.

19. $2021 \mathrm{RDG}$. Correlation of the units (U) of the Elecsys ${ }^{\circledR}$ Anti-SARS-CoV-2 S assay to the "binding antibody units" (BAU) of the first WHO International Standard for anti-SARS-CoV-2 immunoglobulin. Penzberg, Germany.

20. WHO. WHO. First WHO International Standard for anti-SARS-CoV-2 Immunoglobulin. . https://cdn.who.int/media/docs/default-source/biologicals/ecbs/bs2020-2403-sars-cov-2-ab-ik-17-nov-2020 4ef4fdae-e1ce-4ba7-b21ad725c68b152b.pdf?sfvrsn=662b46ae_8\&download=true. Accessed $9^{\text {th }}$ October 2021.

21. Marfella R, D'Onofrio N, Sardu C, et al. Does poor glycaemic control affect the immunogenicity of the COVID-19 vaccination in patients with type 2 diabetes: The CAVEAT study. Diabetes Obes Metab. 2021.

22. Patel EU, Bloch EM, Clarke W, et al. Comparative Performance of Five Commercially Available Serologic Assays To Detect Antibodies to SARS-CoV-2 and Identify Individuals with High Neutralizing Titers. J Clin Microbiol. 2021;59(2).

23. Yun S, Ryu JH, Jang JH, et al. Comparison of SARS-CoV-2 Antibody Responses and Seroconversion in COVID-19 Patients Using Twelve Commercial Immunoassays. Ann Lab Med. 2021;41(6):577-587.

24. Zimmermann P, Curtis N. Factors That Influence the Immune Response to Vaccination. Clin Microbiol Rev. 2019;32(2).

25. Ma BM, Yap DYH, Yip TPS, Hung IFN, Tang SCW, Chan TM. Vaccination in patients with chronic kidney disease-Review of current recommendations and recent advances. Nephrology (Carlton). 2021;26(1):5-11.

26. Van der Wielen M, Van Damme P, Chlibek R, Smetana J, von Sonnenburg F. Hepatitis A/B vaccination of adults over 40 years old: comparison of three vaccine regimens and effect of influencing factors. Vaccine. 2006;24(26):5509-5515.

27. Khoury DS, Cromer D, Reynaldi A, et al. Neutralizing antibody levels are highly predictive of immune protection from symptomatic SARS-CoV-2 infection. Nat Med. 2021;27(7):1205-1211.

28. Suhandynata RT, Hoffman MA, Huang D, et al. Commercial Serology Assays Predict Neutralization Activity against SARS-CoV-2. Clin Chem. 2021;67(2):404-414. 
Table 1.

Baseline Characteristics $(\mathrm{N}=150)$

\begin{tabular}{|c|c|c|c|c|c|c|}
\hline \multirow[t]{2}{*}{ Variables } & All & $\begin{array}{c}\text { T1DM - } \\
\text { insufficiently } \\
\text { controlled }\end{array}$ & $\begin{array}{c}\text { T1DM - } \\
\text { well } \\
\text { controlled }\end{array}$ & $\begin{array}{c}\text { T2DM - } \\
\text { insufficiently } \\
\text { controlled }\end{array}$ & $\begin{array}{c}\text { T2DM - } \\
\text { well } \\
\text { controlled }\end{array}$ & \multirow[t]{2}{*}{$\begin{array}{c}\mathrm{P}- \\
\text { value }\end{array}$} \\
\hline & $(\mathrm{N}=150)$ & $(\mathrm{N}=26)$ & $(\mathrm{N}=49)$ & $(\mathrm{N}=38)$ & $(\mathrm{N}=37)$ & \\
\hline Age & $49.2 \pm 14.5$ & $42.7 \pm 14.0$ & $40.8 \pm 14.8$ & $56.9 \pm 9.6$ & $56.3 \pm 10.3$ & $<0.001$ \\
\hline \multicolumn{7}{|l|}{ Sex, n (\%) } \\
\hline Female & $68(45.3)$ & $10(38.5)$ & $24(49.0)$ & $20(52.6)$ & $14(37.8)$ & \multirow[t]{2}{*}{0.491} \\
\hline Male & $82(54.7)$ & $16(61.5)$ & $25(51.0)$ & $18(47.4)$ & $23(62.2)$ & \\
\hline \multicolumn{7}{|l|}{ Vaccine, n (\%) } \\
\hline BioNTech Pfizer & $129(86.0)$ & $24(92.2)$ & $40(81.6)$ & $35(92.1)$ & $30(81.1)$ & \multirow[t]{3}{*}{0.542} \\
\hline Moderna & $13(8.7)$ & $1(3.9)$ & $5(10.2)$ & $3(0.0)$ & $4(10.8)$ & \\
\hline AstraZeneca & $8(5.3)$ & $1(3.9)$ & $4(8.2)$ & $0(7.9)$ & $3(8.1)$ & \\
\hline Duration of diabetes & $16.0 \pm 12.0$ & $23.6 \pm 13.6$ & $18.1 \pm 12.9$ & $13.5 \pm 9.4$ & $10.6 \pm 8.5$ & $<0.001$ \\
\hline Insulin therapy & $104(69.3)$ & $26(100.0)$ & $48(98.0)^{*}$ & $21(55.3)$ & $9(24.3)$ & $<0.001$ \\
\hline \multicolumn{7}{|l|}{ Comorbidity } \\
\hline Hypertension, n (\%) & $66(44.0)$ & $7(26.9)$ & $9(18.4)$ & $25(65.8)$ & $25(67.6)$ & $<0.001$ \\
\hline Coronary Heart Disease, n (\%) & $14(9.3)$ & $1(3.9)$ & $2(4.1)$ & $6(15.8)$ & $5(13.5)$ & 0.160 \\
\hline Myocardial Infarction, n (\%) & $7(4.7)$ & $0(0.0)$ & $1(2.0)$ & $4(10.5)$ & $2(5.4)$ & 0.209 \\
\hline Stroke, n (\%) & $5(3.3)$ & $1(3.9)$ & $0(0.0)$ & $3(7.9)$ & $1(2.7)$ & 0.169 \\
\hline Heart Failure, n (\%) & $4(2.7)$ & $0(0.0)$ & $1(2.0)$ & $2(5.3)$ & $1(2.7)$ & 0.830 \\
\hline PAD, $\mathrm{n}(\%)$ & $10(6.7)$ & $0(0.0)$ & $2(4.1)$ & $4(10.5)$ & $4(11.1)$ & 0.232 \\
\hline PTCA/CABG, n (\%) & $7(4.7)$ & $0(0.0)$ & $1(2.0)$ & $3(7.9)$ & $3(8.1)$ & 0.327 \\
\hline CVD, $\mathrm{n}(\%)$ & $15(10.0)$ & $1(3.9)$ & $2(4.1)$ & $6(15.8)$ & $6(16.2)$ & 0.111 \\
\hline Hyperlipidemia, n (\%) & $70(46.7)$ & $11(42.3)$ & $12(24.5)$ & $25(65.8)$ & $22(59.5)$ & $<0.001$ \\
\hline Liver disease, $\mathrm{n}(\%)$ & $23(15.3)$ & $1(3.9)$ & $1(2.0)$ & $9(23.7)$ & $12(32.4)$ & $<0.001$ \\
\hline History of cancer, n (\%) & $8(5.3)$ & $2(7.7)$ & $0(0.0)$ & $2(5.3)$ & $4(10.8)$ & 0.083 \\
\hline \multicolumn{7}{|l|}{ Microvascular complications } \\
\hline Retinopathy, n (\%) & $21(14.0)$ & $7(26.9)$ & $6(12.2)$ & $5(13.2)$ & $3(8.1)$ & 0.215 \\
\hline $\begin{array}{l}\text { Polyneuropathy, n (\%) } \\
\text { Labs }\end{array}$ & $29(19.3)$ & $3(11.5)$ & $3(6.1)$ & $15(39.5)$ & $8(21.6)$ & 0.001 \\
\hline $\mathrm{HbA} 1 \mathrm{c}(\mathrm{mmol} / \mathrm{mol})$ & $56.7 \pm 12.5$ & $67.9 \pm 9.8$ & \multirow{2}{*}{$\begin{array}{c}49.3 \pm 6.7 \\
101.5 \\
\pm 17.5\end{array}$} & $67.8 \pm 9.3$ & $47.5 \pm 6.9$ & $<0.001$ \\
\hline eGFR $\left(\mathrm{ml} / \mathrm{min} / 1.73 \mathrm{~m}^{2}\right)$ & $92.5 \pm 20.9$ & $96.3 \pm 26.5$ & & $80.6 \pm 18.9$ & $89.6 \pm 15.9$ & $<0.001$ \\
\hline $\begin{array}{l}\mathrm{HDL}-\mathrm{C}(\mathrm{mg} / \mathrm{dl}) \\
\mathrm{LDL}-\mathrm{C}(\mathrm{mg} / \mathrm{dl})\end{array}$ & $\begin{array}{l}58.5 \pm 19.5 \\
91.4 \pm 34.2\end{array}$ & $\begin{array}{l}63.1 \pm 22.2 \\
95.0 \pm 32.6\end{array}$ & $\begin{array}{l}69.1 \pm 17.1 \\
93.8 \pm 29.1\end{array}$ & $\begin{array}{l}47.3 \pm 13.3 \\
85.1 \pm 36.7\end{array}$ & $\begin{array}{l}52.9 \pm 18.1 \\
91.9 \pm 39.3\end{array}$ & $\begin{array}{c}<0.001 \\
0.633\end{array}$ \\
\hline Triglyceride (mg/dl) & $115.1 \pm 99.4$ & $99.2 \pm 46.3$ & $\begin{array}{r}175.6 \\
\pm 164.4\end{array}$ & $78.3 \pm 30.7$ & $113.4 \pm 64.6$ & 0.121 \\
\hline
\end{tabular}

Qualitative variables are presented as frequencies and percentages (\%).

Quantitative variables are presented as means and standard deviations $\pm S D$.

Chi-square or Fischer's exact tests were applied to compare qualitative variables with diabetes groups. One-way ANOVA tests were applied to compare quantitative variables with diabetes groups. PAD=peripheral artery disease; PTCA=percutaneous transluminal coronary angiography, $\mathrm{CABG}=$ coronary artery bypass graft;

CVD=cardiovascular disease; HDL-C=high-density-lipoprotein-cholesterol; LDL$\mathrm{C}=$ low-density-lipoprotein-cholesterol; *one participant was during the honeymoon period and off insulin at the time of enrollment 


\section{Figure legends:}

\section{Figure 1}

Side effects after vaccination, overall and by types of diabetes A: after vaccination 1, B: after vaccination 2

\section{Figure 2}

A Comparison of anti-SARS-CoV-2-S antibodies between diabetes and healthy controls after second vaccination.

B Comparison of anti-SARS-CoV-2-S antibodies in people with well and insufficiently controlled type 1 and type 2 diabetes

$\mathrm{P}$-values are adjusted for age and sex using quantile regression and for multiple comparison using Bonferroni correction.

\section{Figure 3}

Correlation plots for selected clinical characteristics

$r$ : Pearson Correlation Coefficient, $p$ : $p$-value for Pearson Correlation 


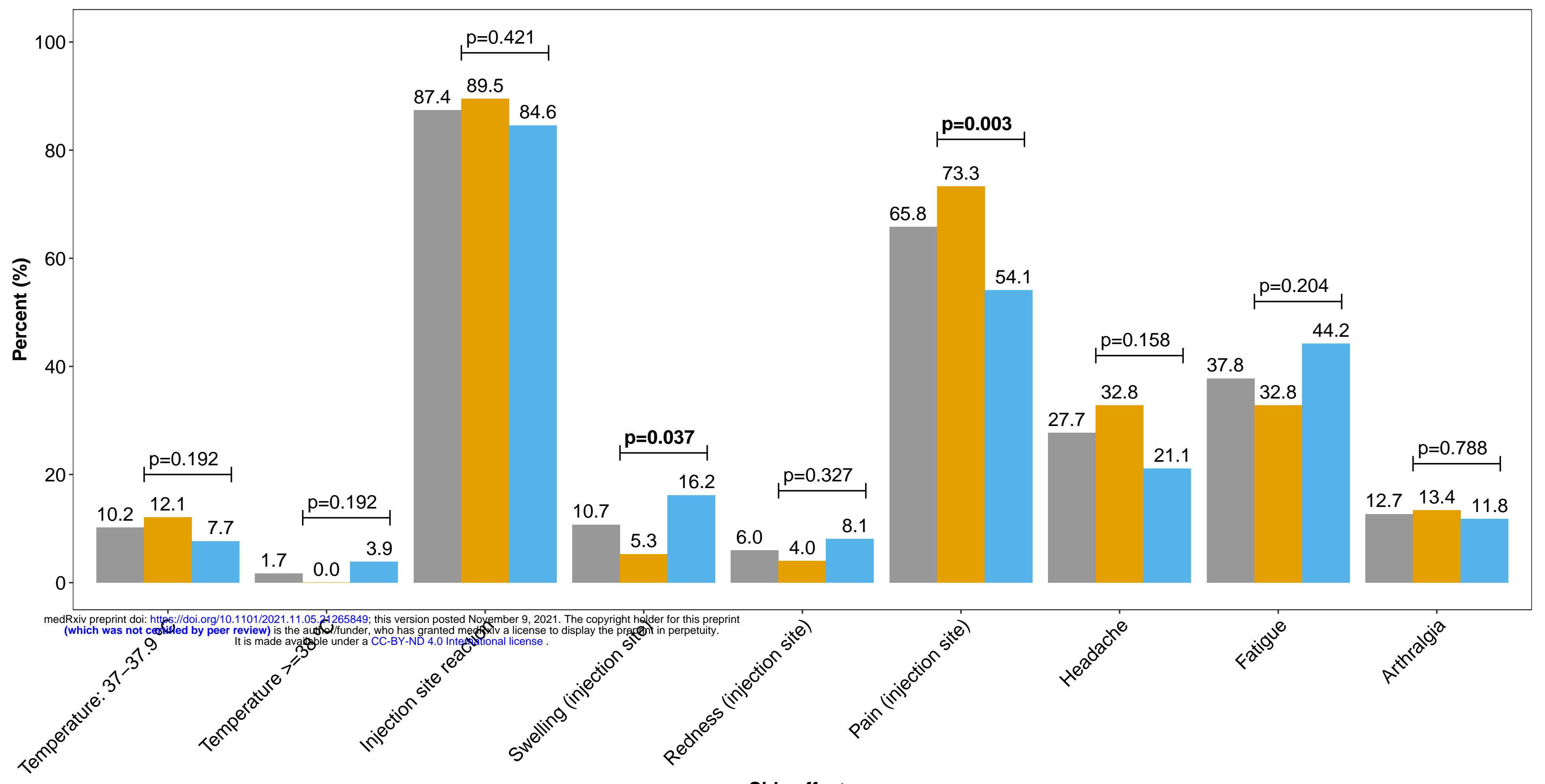

B

Side effects

All T1DM T2DM

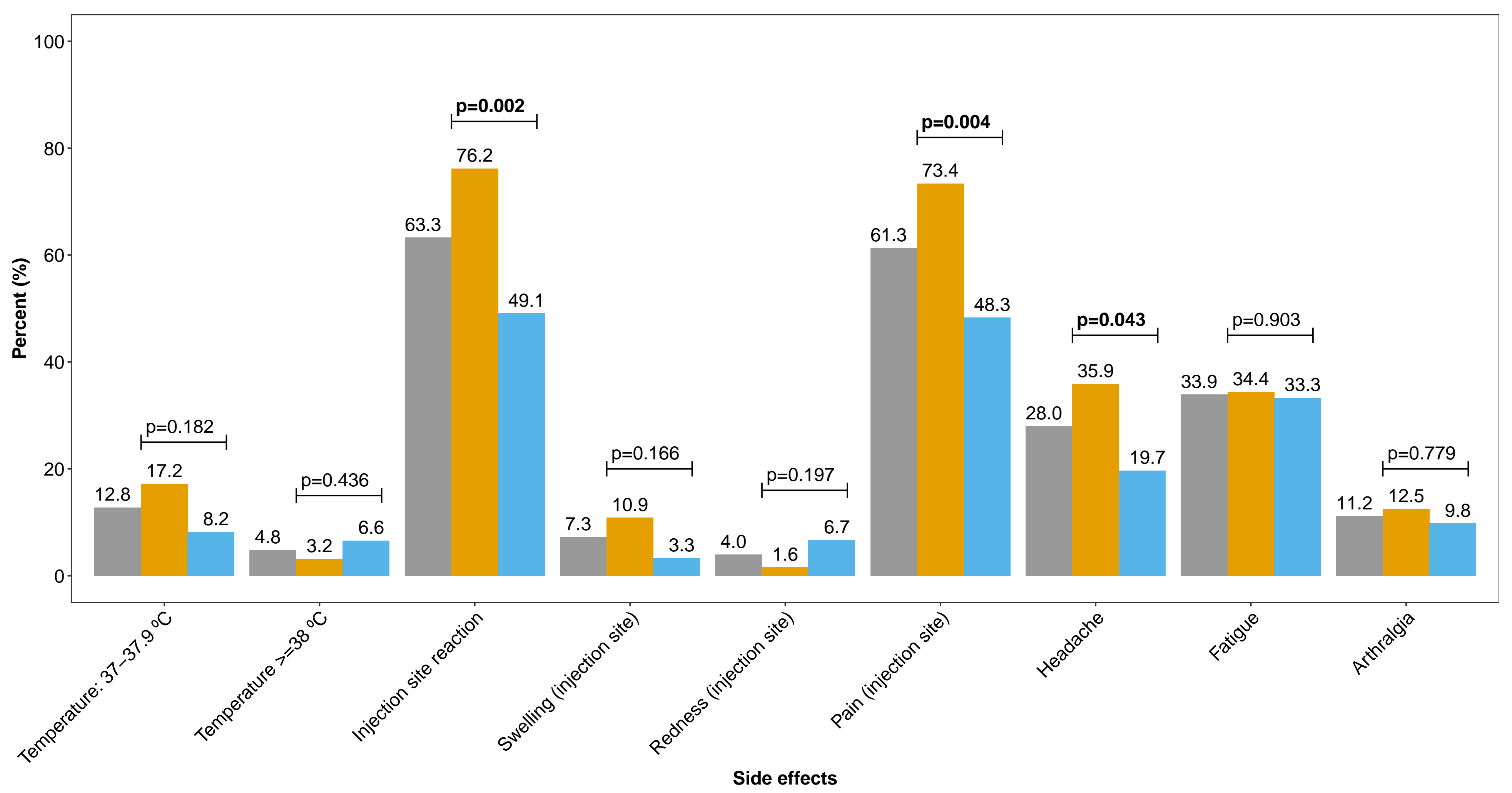


A

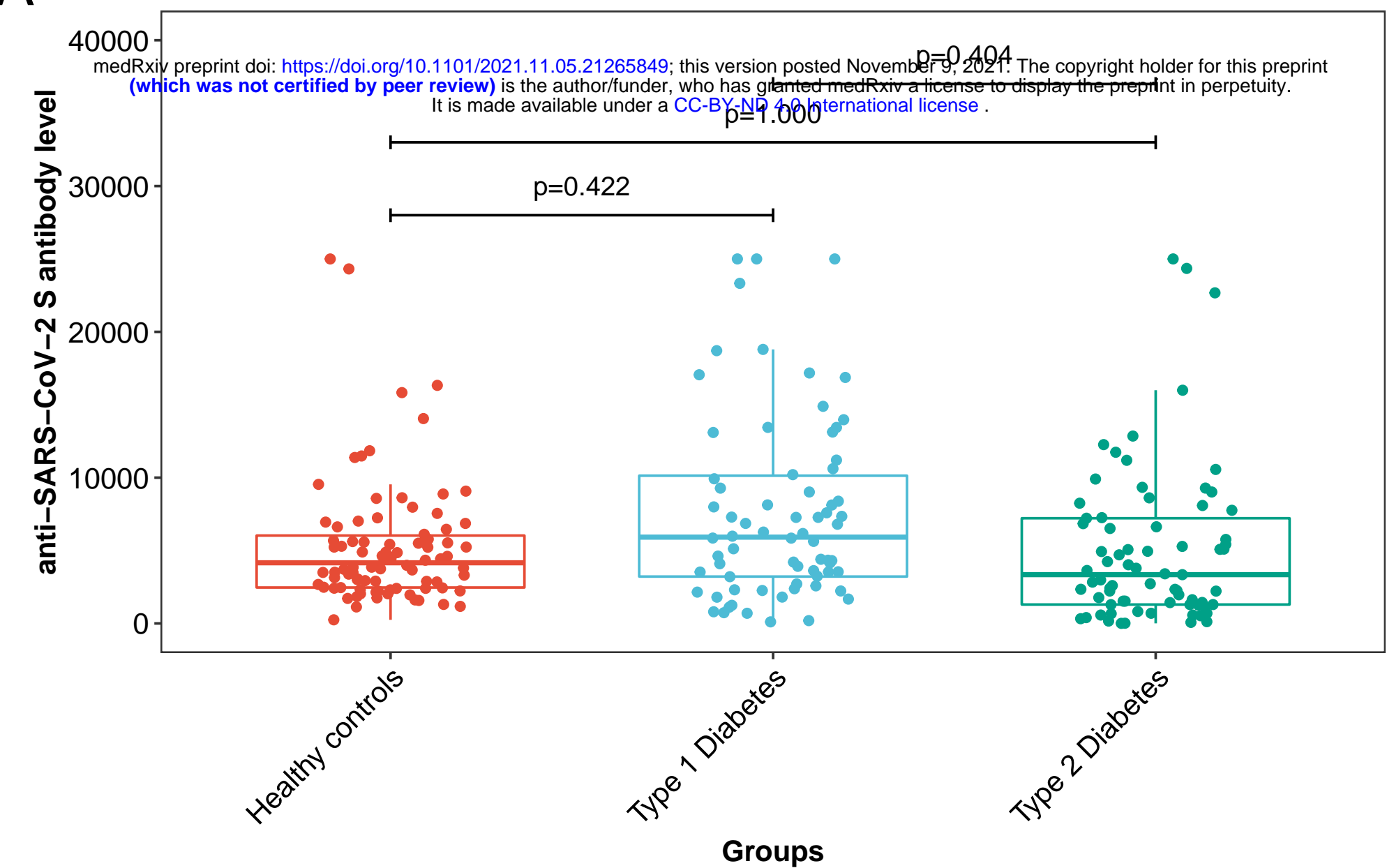

B

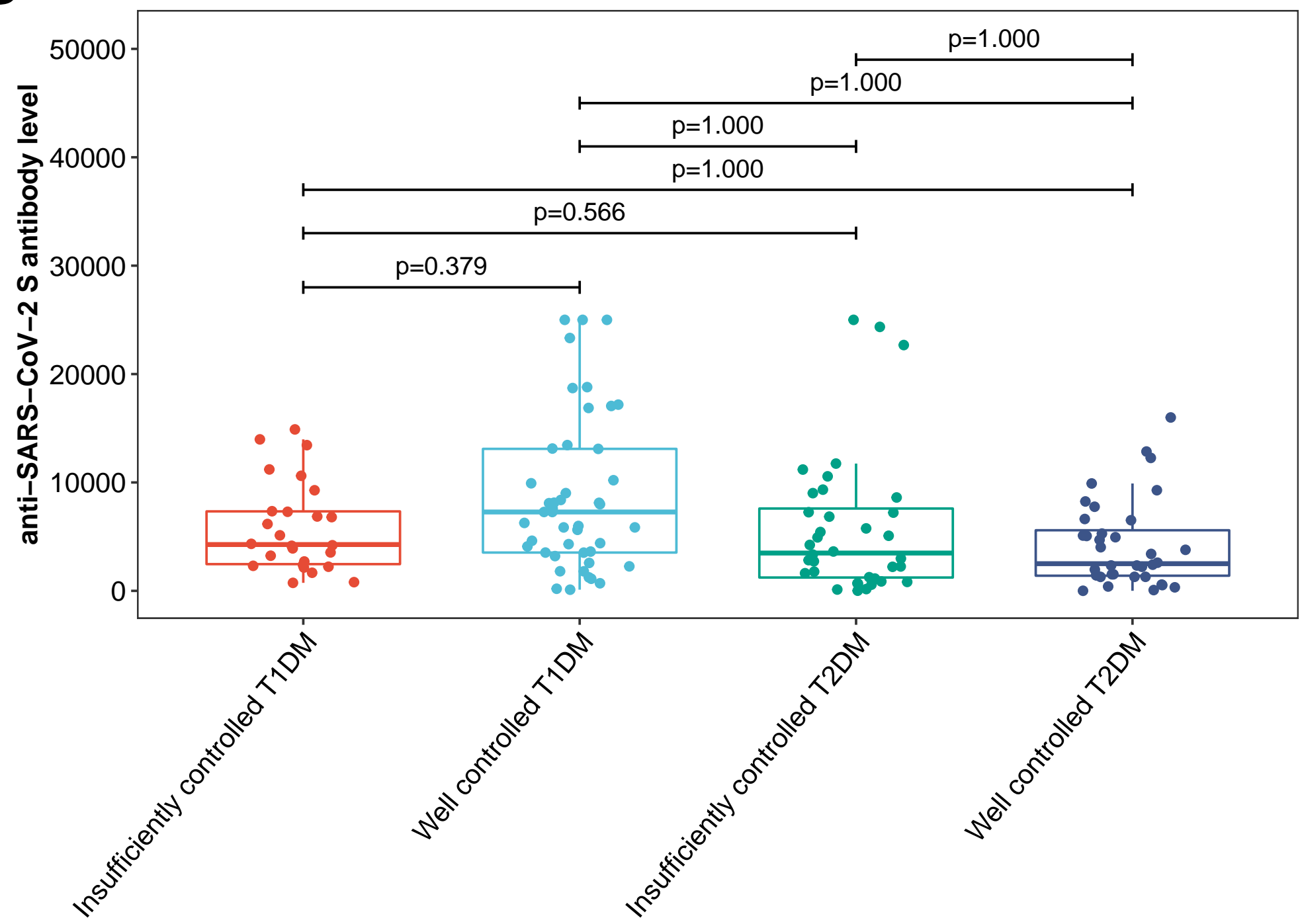



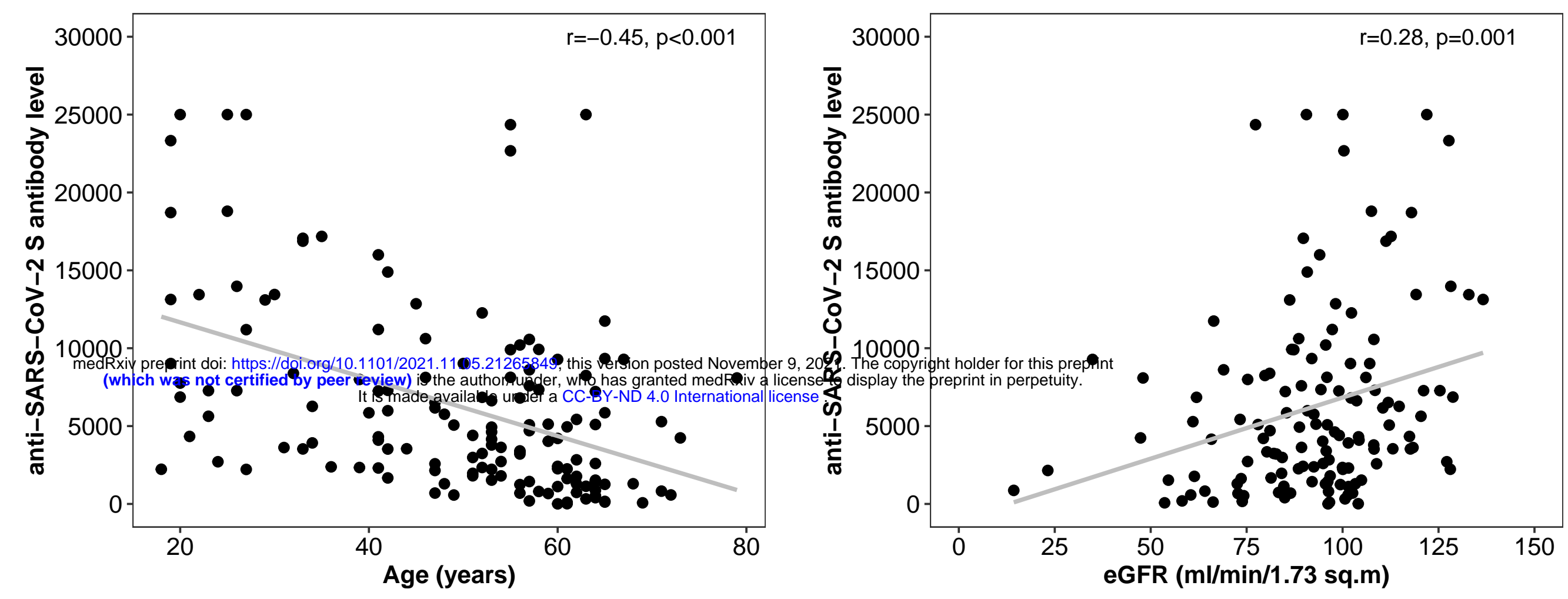

C
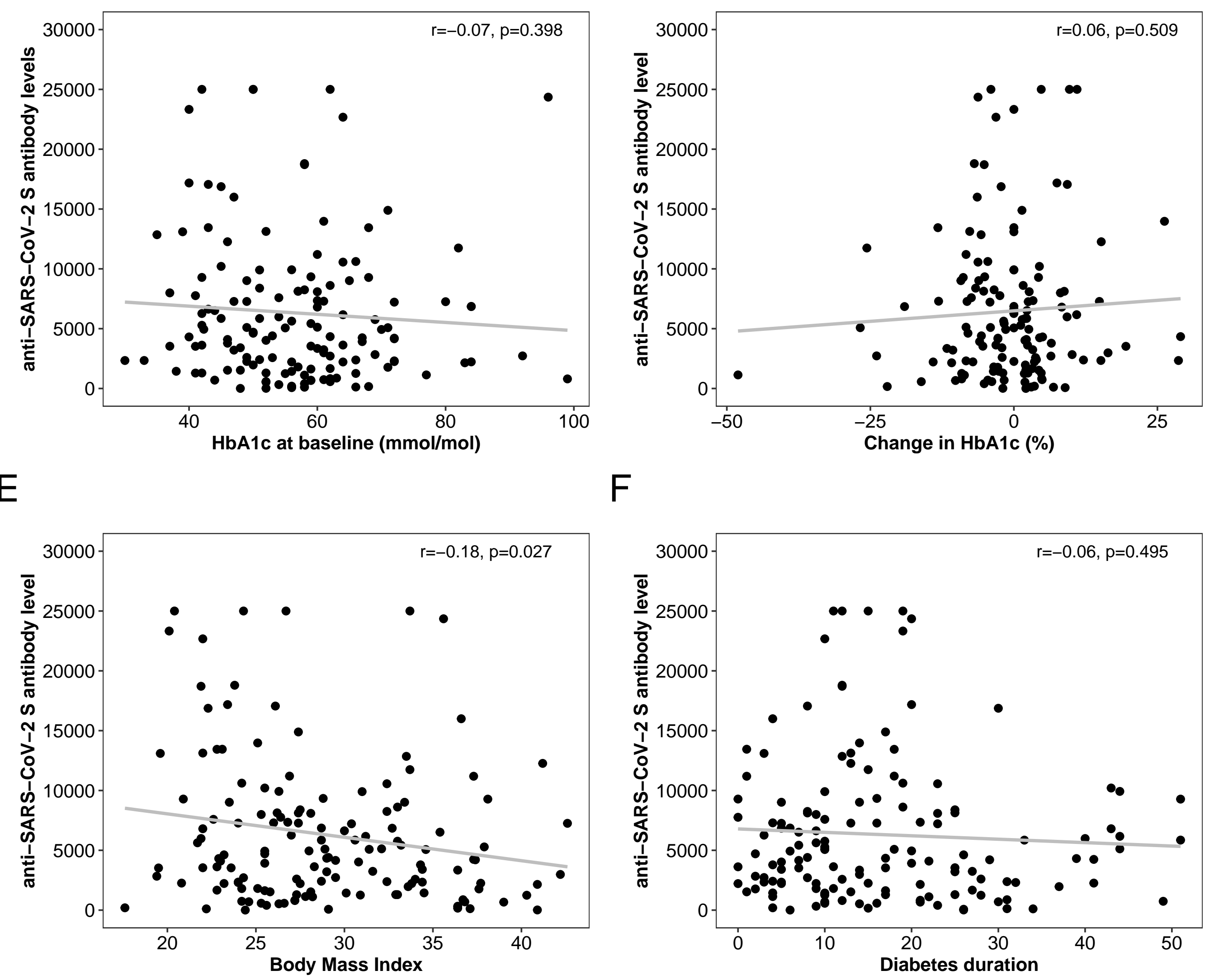